\title{
Temperature-Dependent Excited State Absorption in DNA and LNA Oligomers Supports an Emerging Model of Excited State Dynamics in DNA
}

\author{
Stanislav O. Konorov ${ }^{1,2}$, H. Georg Schulze ${ }^{1}$, Christopher J. Addison ${ }^{1,2}$, Charles A. Haynes ${ }^{1,3}$, \\ Robin F.B. Turner ${ }^{*}, 1,2,4$ and Michael W. Blades ${ }^{*}, 2$
}

\author{
${ }^{I}$ Michael Smith Laboratories, The University of British Columbia, 2185 East Mall, Vancouver, BC, V6T 1Z4, Canada \\ ${ }^{2}$ Department of Chemistry, The University of British Columbia, 2036 Main Mall, Vancouver, BC, V6T 1Z1, Canada \\ ${ }^{3}$ Department of Chemical and Biological Engineering, The University of British Columbia, 2360 East Mall, Vancouver, \\ $B C$, V6T 1Z3, Canada \\ ${ }^{4}$ Department of Electrical and Computer Engineering, The University of British Columbia, 2332 Main Mall, Vancouver, \\ $B C$, V6T 1Z4, Canada
}

\begin{abstract}
Transient absorption measurements of excited states in DNA and LNA were performed using a femtosecond pump-probe arrangement with excitation at $266 \mathrm{~nm}$ and absorption monitored at $400 \mathrm{~nm}$ while varying the sample temperature between $5{ }^{\circ} \mathrm{C}$ and $70{ }^{\circ} \mathrm{C}$. Samples consisted of adenine monophosphate monomer, polyadenine 12-mer in singlestranded form, and polyadenine 12-mer in hybridized form. Excited states decayed in a biphasic manner with short-lived $\left(\tau_{1}\right)$ and long-lived $\left(\tau_{2}\right)$ components, while the monomer had only a 'single' short-lived decay time. Temperature increases increased absorption intensities and reduced $\tau_{1}$ until they approached those of the monomer at high temperatures (where stacking is minimal). These results suggest that the initial excitation in stacked regions is cooperative and involves several bases and that the number of bases involved is reduced with increasing temperature. In contrast, increasing temperatures had little effect on $\tau_{2}$ while absorption intensities decreased, suggesting that very few, perhaps only two, stacked bases are involved and that their number is reduced at higher temperatures. We found no clear evidence of melting point transitions indicating that those excited states probed with our arrangement were not dependent on base pairing. Our results are consistent with and strengthen an emerging consensus model of excited state dynamics in DNA wherein a UV photon is absorbed collectively by electronically coupled and thus well-stacked intrachain bases. This collective excitation results in a Frenkel exciton that is delocalized over these bases, and the Frenkel exciton then decays rapidly to a long-lived, lower energy, dark intrachain exciplex.
\end{abstract}

Keywords: Transient absorption, locked nucleic acids, excited state dynamics, base stacking, cooperative excitation, Frenkel exciton, charge transfer exciton.

\section{INTRODUCTION}

The canonical B-form DNA molecule has a deceptively simple structural organization. Along one dimension, structural alignment and stability are provided by forces resulting from the base-matching dipole-dipole interactions of Watson-Crick hydrogen bonds [1]. Along the other dimension, base stacking provides structural alignment and stability, but here induced dipole-induced dipole (dispersion) forces are relatively more important [2], especially between adjacent adenines [3]. Insult from a variety of sources, including organic carcinogens [4], oxidants [5], cross-linking agents [6, 7], viruses [8], and radiation [9-11], can cause disarray in this structural organization leading to transcriptional or replication failure. The prevalence of solar radiation and the consequent exposure of genetic material to potentially damaging ultraviolet (UV) rays [10] have generated considerable

*Address correspondence to these authors at the Department of Chemistry, The University of British Columbia, 2036 Main Mall, Vancouver, BC, V6T 1Z1,Canada; E-mails: turner@msl.ubc.ca; blades@chem.ubc.ca interest in this source of insult and its mechanisms of damage, protection, and repair [10-13].

Despite its relatively simple structural organization, the electronic properties of DNA are surprisingly rich, giving rise to numerous phenomena that are not yet well integrated into a comprehensive and coherent view. Previous and present studies seek clarity on the nature of the ground- and excited-state electronic properties of individual bases [1418], and the modifications brought about by pairing [19], stacking [2, 20-30], and hydration [31]. Three specific questions of interest are the following. Are Franck-Condon (FC) excited states localized to a single base or is the initial excitation cooperative? To what degree do excitations have or acquire charge-transfer (CT; excimer) character as opposed to charge-distribution (Frenkel) character? What is the relative importance of base stacking compared to base pairing in governing excited state dynamics?

Currently, we believe that views about the response of DNA to UV excitation are converging on a consensus model of excited state dynamics in DNA, key aspects of which are 
depicted in Fig. (1). In this view, DNA bases reside in regions where they are either well stacked or poorly stacked [32]. In poorly stacked regions, UV light excites bases individually, but in well-stacked regions the bases are electronically coupled and their excitation is collective $[32,33]$. This collective excitation produces a Frenkel exciton distributed over several bases $[22,32,34]$ that quickly $\left(\tau_{1}\right)$ relaxes to lower energy excited states with charge transfer character [22], ultimately yielding long-lived $\left(\tau_{2}\right)$ dark excimers and exciplexes $[34,35]$. It is of interest that the consensus view is rather quiet about the role of base-pairing despite the fact that the contribution of base-pairing to the excited state dynamics in DNA is explicitly questioned [36]. We take this absence, along with experimental evidence favoring stacking over pairing [20], to indicate that base-pairing is not considered to be of major importance in excited state dynamics in DNA.

If the consensus model is correct, one should expect an increase in thermally-induced structural fluctuations to produce a more rapid decline in $\tau_{1}$ than in $\tau_{2}$ since the probability of finding regions with more than two well-stacked bases, available for cooperative excitation, will decline more rapidly with increasing temperature than the probability of finding pairs of adjacent well-stacked bases. Thermal structural fluctuations within a given conformation have been shown by Bouvier, et al. [37] to reduce mixing of monomer excited states between individual bases, hence the spatial extent of the excitons and their lifetimes are reduced. On the other hand, if the initial excitation is localized on a single base, stacking disarray should not have a material effect on the number of excitations or their phenomenological description. Finally, if base-pairing materially influenced the excited state dynamics in base stacks, differences in excited state properties obtained before and after the melting point ought to be noticeable; in the absence of such differences, one may conclude that stacking interactions have greater importance than base pair interactions in the excited state dynamics of adenine stacks.

To test the model outlined above, we investigated the excited state dynamics of single- and double-stranded adenine homopolymers as a function of stacking. Temperature increases were used to manipulate the amount of stacking while following excited state dynamics with transient absorption measurements. Thus a range of ordered structures was interrogated, ranging from relatively ordered to relatively disordered (i.e. corresponding to temperatures below and above the melting point of the double stranded samples, respectively). Briefly, we found that temperature increases resulted in a reduction of the short excited state lifetimes $\left(\tau_{1}\right)$ until they approached monomer values while the absorption intensities increased. The longer excited state lifetimes $\left(\tau_{2}\right)$ remained little altered, but their absorption intensities decreased. The transition through the melting point did not have a marked effect on the results; neither did conformation produce qualitative differences (although the excited state lifetimes in A-form were shorter than those in B-form). Taken together, the results show that the FC state is cooperative and extends over more than two bases and it evolves to a final state, probably a CT exciton, that consists of only two bases. There is little evidence of a melting transition indicating that intrachain base stacking is the dominant factor governing excited state dynamics in adenine stacks. Our results therefore support the emerging consensus model of excited state dynamics in DNA.

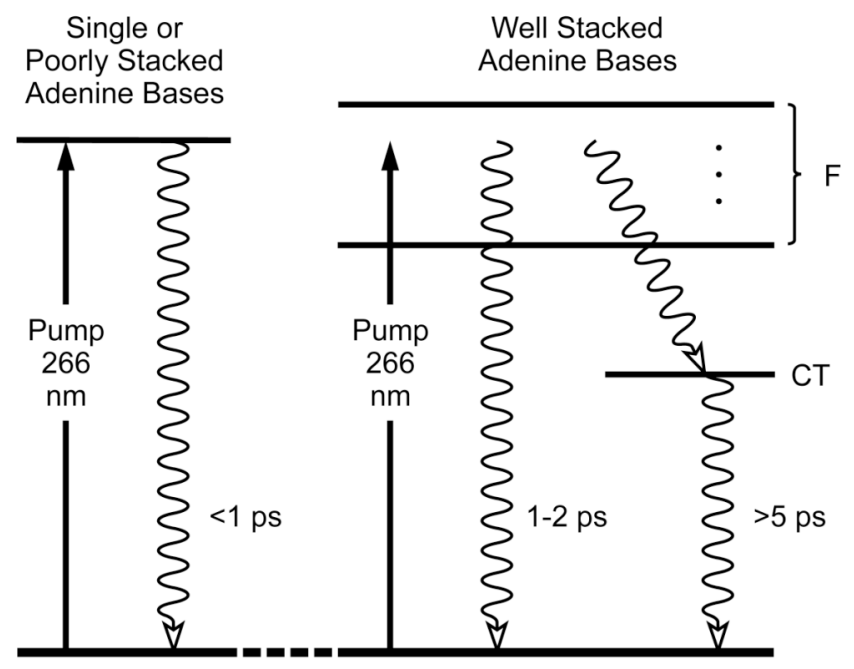

Fig. (1). Energy level diagram showing simplified relaxation pathways and excited state lifetimes. The energy levels on the left side reflect a base under conditions where neighboring adenines are poorly stacked, thus a monomer-like electronic structure prevails; the energy levels on the right side reflect the same base in the same oligomer, but under conditions where neighboring adenines are well stacked and can accommodate delocalized exciton formation (Frenkel exciton (F) and charge transfer exciton (CT)).

\section{MATERIALS AND METHODS}

\section{Oligomer Sample Preparation}

All samples used in this study are defined in Table 1. Sample 1 (Sigma-Aldrich) and samples 2-5 (unpurified from IDT, Coralville, USA) were used to investigate temperature effects in both single- and double-stranded oligomers. We follow here the convention [38] of denoting a monomer or an oligomer containing one or more locked monomers as a LNA; only adenines were locked.

Monomers, single-stranded DNA, and single-stranded LNA were dialyzed in $10 \mathrm{mM}$ phosphate buffer $(\mathrm{pH} \mathrm{6}$ ). Stock concentrations of single-stranded oligomers were determined by measuring the absorbance at $260 \mathrm{~nm}$ and $80{ }^{\circ} \mathrm{C}$ using a Cary 1E UV-vis spectrophotometer (Varian, Palo Alto, CA). Double-stranded samples were prepared by combining 1:1 molar equivalents in vials at $25{ }^{\circ} \mathrm{C}$. The 0.036 $\mathrm{mg} / \mathrm{ml}(25 \mu \mathrm{M})$ oligomers in $10 \mathrm{mM}$ phosphate buffer and $\mathrm{pH} 6$ were divided into $400 \mu \mathrm{L}$ aliquots. Then doublestranded aliquots of 12-mer AT DNA and LNA oligomers were fully hybridized in a thermal cycler (Cetus 480, Perkin Elmer, Wellesley, MA, USA). Hybridization was performed by heating the oligomers up to $80^{\circ} \mathrm{C}$ and then cooling them down to $20{ }^{\circ} \mathrm{C}$ at a rate of $1{ }^{\circ} \mathrm{C} / \mathrm{min}$. A recording of $\mathrm{UV}$ absorbance at $260 \mathrm{~nm}$ when heating Sample 3 (Table 1) from $15{ }^{\circ} \mathrm{C}$ to $75^{\circ} \mathrm{C}$ provided a melting curve for this sample using a concentration of $5 \mu \mathrm{M}$.

\section{Femtosecond Pump-Probe Measurements}

The pump-probe transient absorption measurements were executed with a femtosecond Ti:Sapphire laser setup 
Table 1. Oligomer Sequences Used; Locked Adenine Bases are Bold-Underlined

\begin{tabular}{|c|c|}
\hline Sample ID & Sequence \\
\hline 1 & $5^{\prime}-\mathrm{A}-3^{\prime}$ \\
\hline 2 & $5^{\prime}-\mathrm{AAA}$ AAA AAA AAA-3' \\
\hline 3 & $\begin{array}{l}5^{\prime-A A A} \text { AAA AAA AAA-3' } \\
3^{\prime} \text {-TTT TTT TTT TTT-5' }\end{array}$ \\
\hline 4 & $5^{\prime}-\mathrm{AAA} \underline{\mathrm{A}} \mathrm{AA} \underline{\mathrm{A} A \mathrm{~A}} \underline{\mathrm{A}} \mathrm{AA}-3^{\prime}$ \\
\hline 5 & $\begin{array}{l}5^{\prime}-\mathrm{AAA} \text { AAA } \text { AAA } \text { AAA-3' } \\
3^{\prime}-\mathrm{TTT} \text { TTT TTT TTT-5' }\end{array}$ \\
\hline
\end{tabular}

(Synergy, Femtolasers, Vienna, Austria; master oscillator and SpitFirePro amplifier, SpectraPhysics, Mountain View, CA, USA). The laser generated $60 \mathrm{fs}$ laser pulses, 2 $\mathrm{mJ} /$ pulse, $1 \mathrm{kHz}$ repetition rate, at a central wavelength of $800 \mathrm{~nm}$. The pump beam employed the third harmonic of the main laser radiation at $266 \mathrm{~nm}$ with about $1 \mathrm{GW} / \mathrm{cm}^{2}$ intensity at the sample which was found to yield the best signalto-noise performance without inducing significant twophoton ionization. The probe beam employed part of the second harmonic $(400 \mathrm{~nm})$ in the third harmonic generation stage. Polarization of the pump and probe beams were oriented at the magic angle with probe and pump beams being collinear. UV radiation was filtered out for probe detection using UV-opaque Plexiglas. Every third pulse of the pump radiation was transmitted using a mechanical chopper. The probe radiation energy was attenuated by at least a factor of 10 compared to the pump radiation. The probe signal was detected with an amplified silicon photodiode (Det 210, Thorlabs, Newton, NJ, USA) connected to a lock-in amplifier (Model SR830 DSP, Stanford Research Systems, Sunnyvale, CA, USA). The time delay between pump and probe was scanned in $67 \mathrm{fs}$ steps and $667 \mathrm{fs}$ as sample absorbance was measured in a quartz cuvette with a $4 \mathrm{~mm}$ optical path length.

\section{Data Analysis}

Data were manipulated and analyzed using Origin (OriginLab, Natick, MA, USA) and MATLAB (The MathWorks, Natick, MA, USA) software running under Windows XP (Microsoft, Redmond, WA). Excited state absorption was used for data analysis as obtained (i.e. not normalized to the maximum value at every temperature). Curve fitting procedures were performed in Origin and consisted of fitting one or two exponential functions to the data. Note that since the melting temperature, $T_{m}$, is concentration dependent [39], a small correction $\left(\sim+4{ }^{\circ} \mathrm{C}\right)$ has to be made to align the UV data to the excited state absorption (ESA) data. However, since this correction is small relative to our temperature resolution, it was not performed.

\section{RESULTS AND DISCUSSION}

The results show the same trends as functions of temperature for all the samples in Table 1, Sample 1, the adenine monomer, excepted. Specifically, the results are qualitatively similar for polyadenine in both single stranded and hybrid- ized DNA configurations. LNAs have much shorter excited state lifetimes than DNA [40], but otherwise LNA samples show qualitative results similar to those of DNA, whether single- or double-stranded. The results for DNA and LNA are therefore not discussed separately in the more detailed analyses below.

\section{Co-Operative Excitation}

For the adenine monomer (Sample 1, Table 1) one fast relaxation time $\left(\tau_{1}, 0.9 \mathrm{ps}\right)$ was observed at all temperatures (Fig. 2). This result shows that there are no significant influences, within experimental error, of the temperature on the electronic level structure in the adenine monomer.

(a)

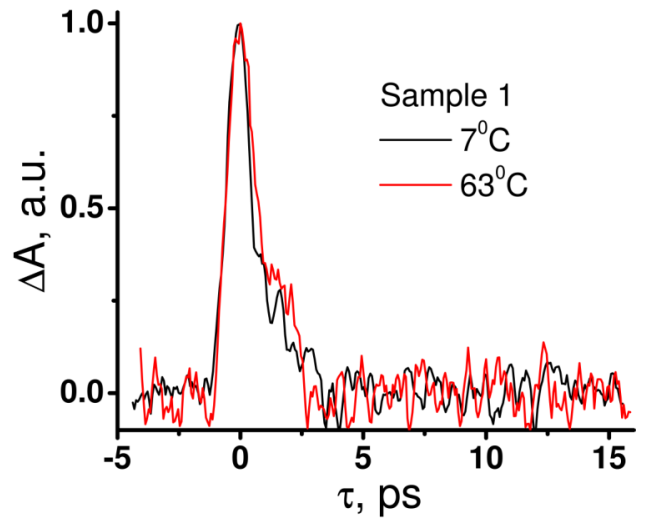

(b)

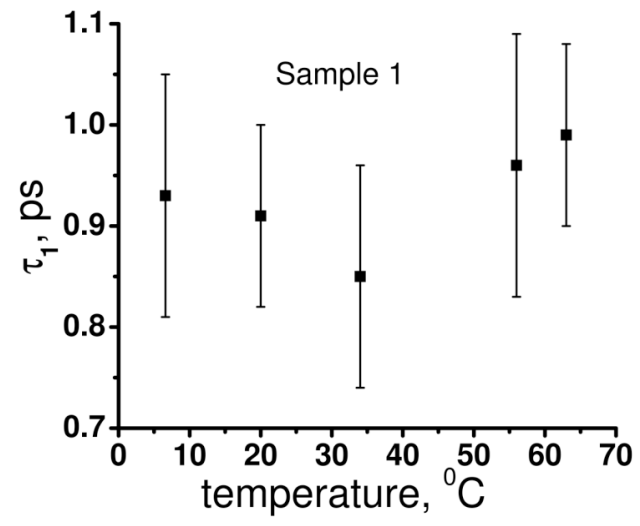

Fig. (2). The excited state absorption intensities (a) and lifetimes (b) of the adenine monomer at different temperatures obtained with a femtosecond experiment using a pump at $266 \mathrm{~nm}$ and probe at $400 \mathrm{~nm}$.

The electronic excited state dynamics are quite different for 12-mer samples (Samples 2-5) as shown in Figs. (3-6). At least two relaxation times $\left(\tau_{1}\right.$, up to a couple of picoseconds) and ( $\tau_{2}$, up to sub- hundred picoseconds) are observed for single and double stranded DNA (Samples 2, 3; Table 1) and LNA (Samples 3, 4; Table 1). The fast component $\left(\tau_{1}\right)$ is the same for both single- and double-stranded DNA, a variant of B-form denoted $\mathrm{B}^{*}[38,41,42]$ (Figs. 4a, 6a). It shortens with increasing temperature from $\sim 1.3 \mathrm{ps}$ at $\sim 10{ }^{\circ} \mathrm{C}$ to $\sim 0.9$ ps at $\sim 60{ }^{\circ} \mathrm{C}$ (i.e. above the melting temperatures of Samples 3 and 5 near $50{ }^{\circ} \mathrm{C}$ ) to a value in line with that of the monomer. The ESA intensities $\left(\mathrm{A}_{1}\right)$, in contrast, increase over the same temperature range (Figs. $4 \mathbf{b}, \mathbf{6 b}$ ). Furthermore, 
these observations are not non-specific, i.e. occurring universally, as discussed for $\left(\tau_{2}\right)$ further below.

(a)

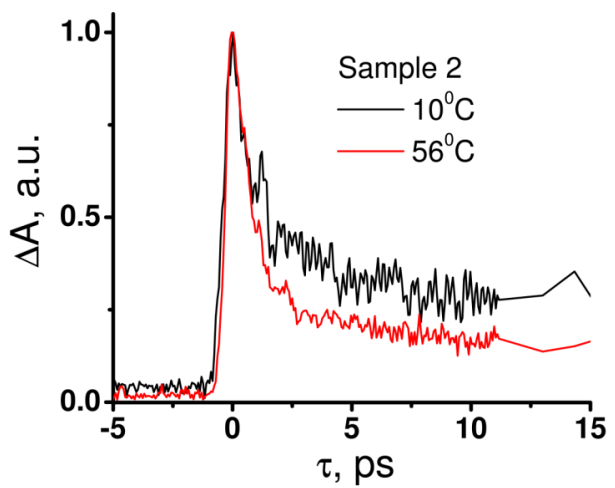

(b)

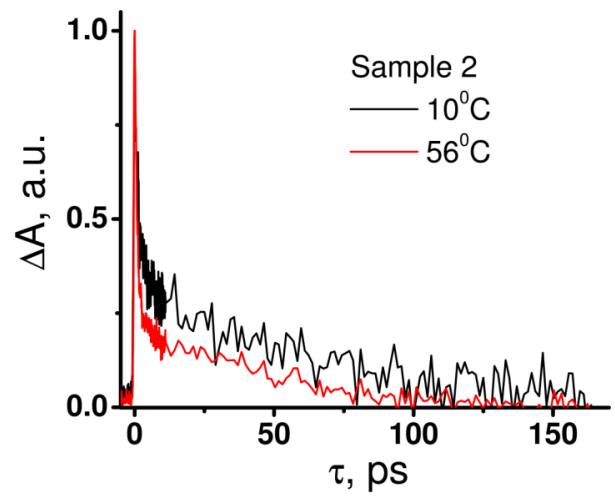

(c)

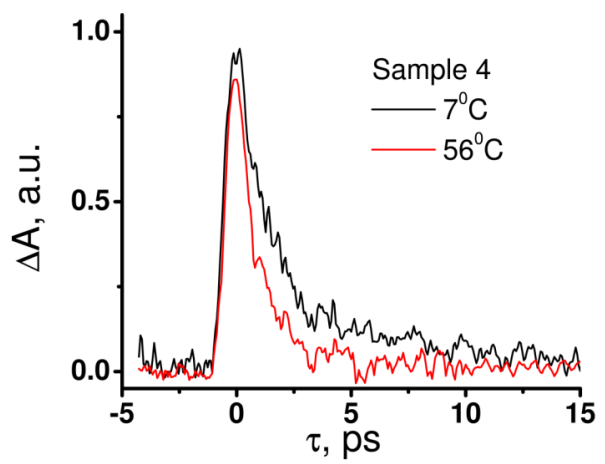

(d)

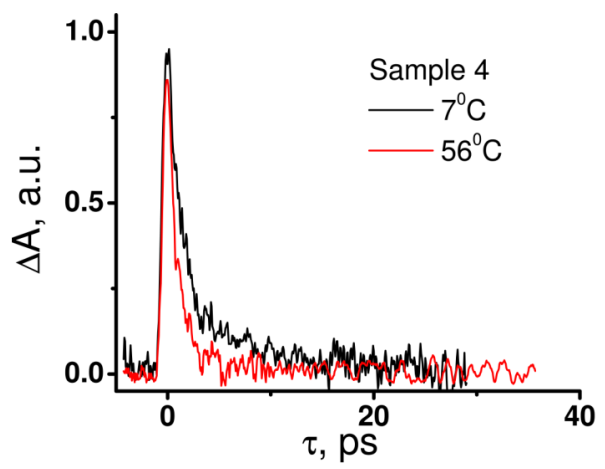

Fig. (3). The excited state absorption intensities at short (a), (c) and longer (b), (d) timescales for Samples 2 and 4, respectively. (a)

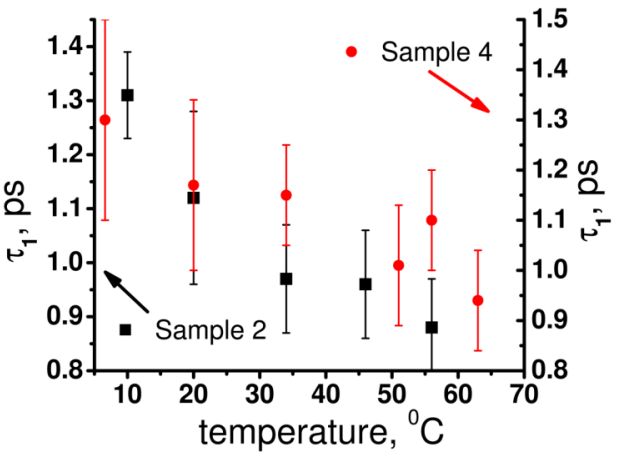

(b)

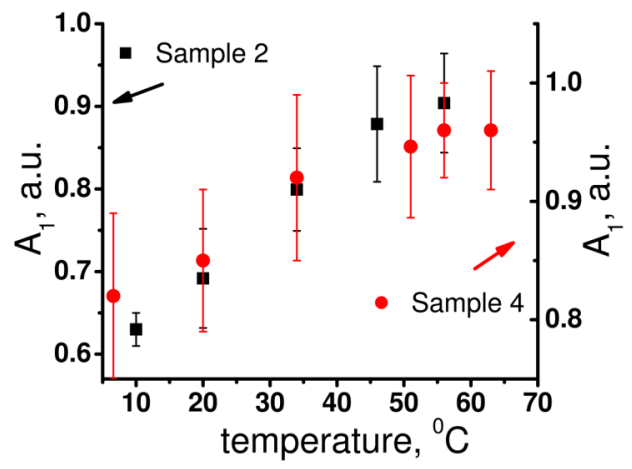

(c)

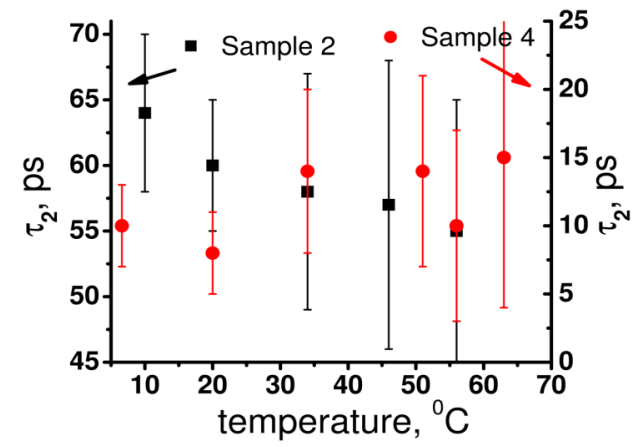

(d)

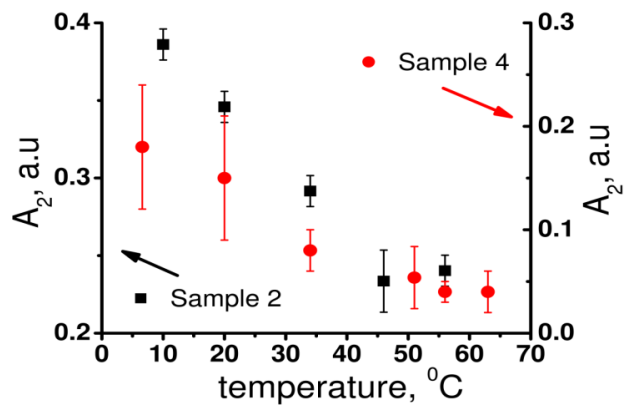

Fig. (4). The short-lived excited state lifetimes (a) and absorption intensities (b), as well as long-lived excited state lifetimes (c) and absorption intensities (d), of Samples 2 and 4 at different temperatures obtained using a femtosecond pump at $266 \mathrm{~nm}$ and femtosecond probe at $400 \mathrm{~nm}$. The short-lived excited states appear to involve several bases since their lifetimes decline with a loss of temperature-dependent stacking, but the long-lived excited states appear unaffected by temperature changes although fewer such states are formed at higher temperatures. 
(a)

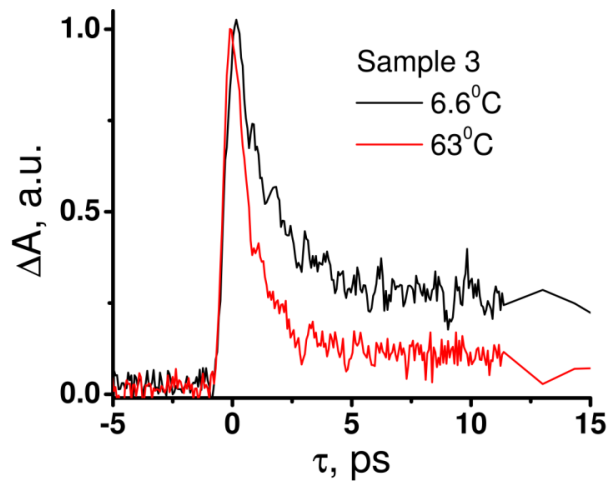

(b)

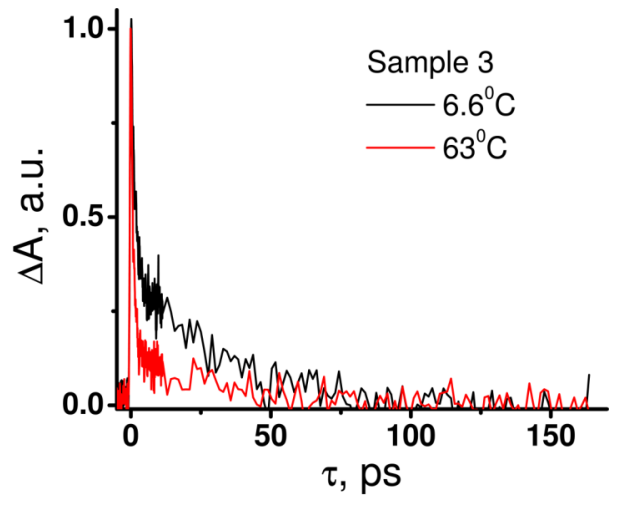

(c)

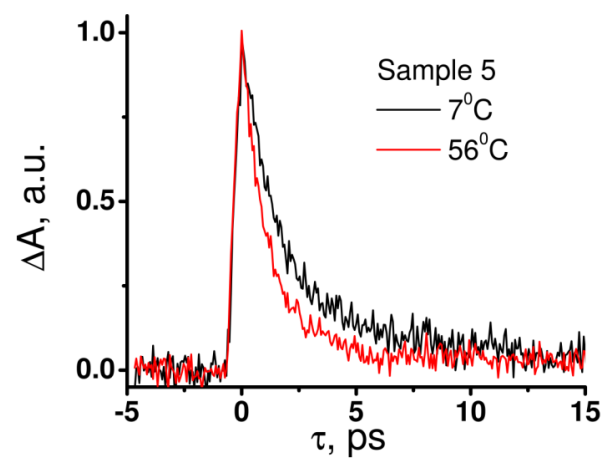

(d)

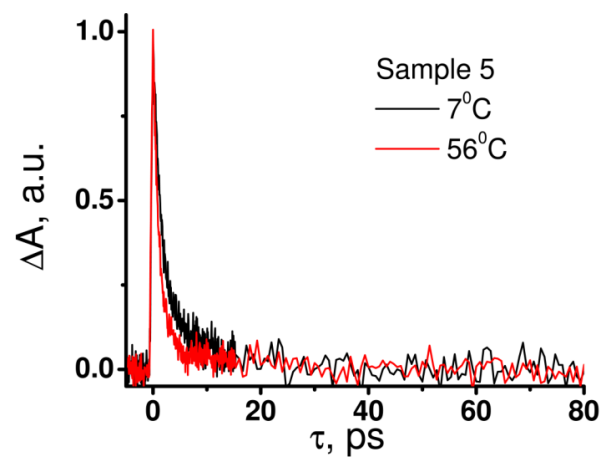

Fig. (5). The excited state absorption intensities at short (a), (c) and longer (b), (d) timescales for Samples 3 and 5, respectively. (a)

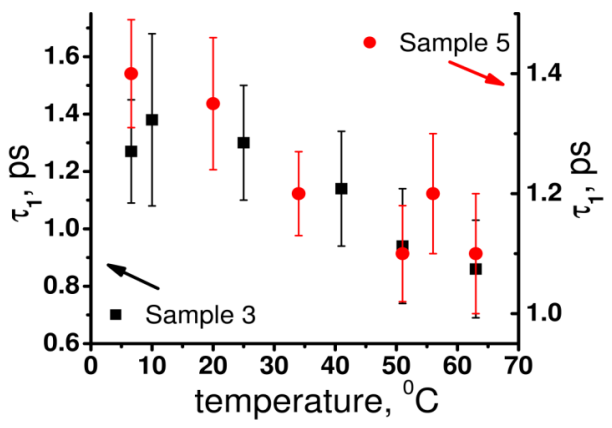

(b)

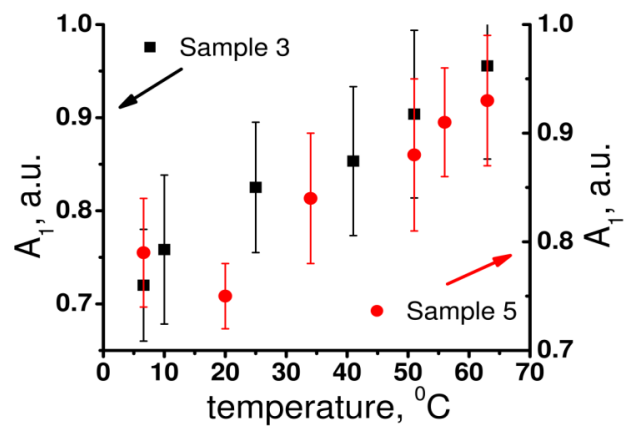

(c)

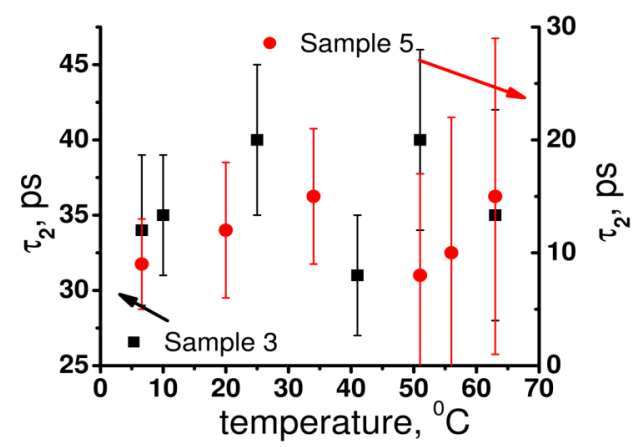

(d)

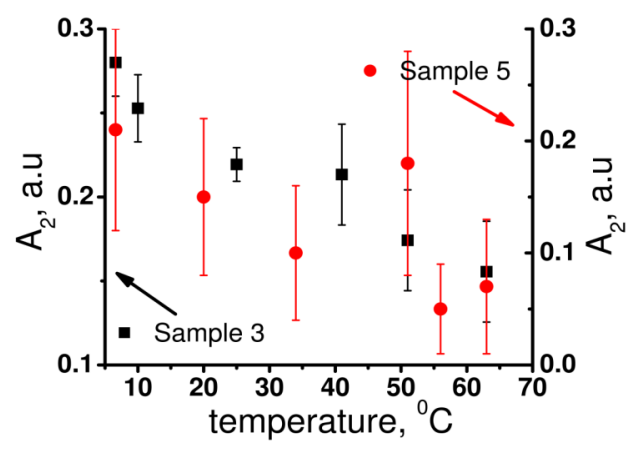

Fig. (6). The short-lived excited state lifetimes (a) and absorption intensities (b), as well as long-lived excited state lifetimes (c) and absorption intensities (d), of Samples 3 and 5 at different temperatures obtained using a femtosecond pump at $266 \mathrm{~nm}$ and femtosecond probe at $400 \mathrm{~nm}$. The results are very similar to those for $\left(\mathrm{dA}_{12}\right)$ indicating that structural order exists in the single strand and that excited state dynamics is dominated by stacking. 
We have previously shown that $\tau_{1}$ generally increases with the number of adenines in the chain [40], thus a reduction in $\tau_{1}$ is consistent with a reduction in the number of stacked adenines. The number of stacked bases is also known to decrease with increasing temperature [43, 44]. Thus, based on the consensus model, one would expect the lifetime of the initial excited state in adenine stacks to change with increasing temperature so that it conforms more to that of the monomer. This occurs because well-stacked domains become smaller and co-operative excitation involves fewer bases (see below), leading to a reduction in $\tau_{1}$. One would also expect the number of single bases available for non-cooperative excitation to increase since more of the bases are now unstacked. Because electronic coupling (i.e. blue shift) $[21,45]$ is now reduced, their excitation threshold is also lowered. Both of the preceding effects would lead to elevated ESA intensities as observed for $\mathrm{A}_{1}$. Co-operative excitation of the $\mathrm{FC}$ state $[32,35]$ receives therefore strong support from these results.

\section{Excited State Character}

The absence of a clear double exponential decay at short time scales (Figs. 3a, 5a) implies that there is no sharp border between unstacked bases with monomer relaxation dynamics and stacked bases with exciton relaxation dynamics. Excitation in stacking domains incorporating a different number of adenines produces excitons distributed over these bases [22, 45], thus each exciton has its own relaxation time depending on the number of bases involved as well as the strength of coupling between these bases. The observed fast relaxation $\left(\tau_{1}\right)$ is therefore a superposition of these different exciton relaxation times. At higher temperatures, fewer bases are stacked [44], thus the stacking domains are smaller and a concomitant reduction of $\tau_{1}$ occurs with temperature (Figs. 4a, 6a), approaching that of the monomer. The general increase in $\tau_{1}$ with oligomer chain length [40] and the reduction in $\tau_{1}$ with increasing temperature, thus with inferred decreasing stack length, clearly demonstrate that the initial excited state is delocalized over several bases. Because Frenkel excitons can migrate coherently [46], in this case, between adjacent adenines [36], delocalization over several bases can occur [22, 45]. Frenkel exciton states should also reflect the singlet excitation energies of the ensembles being cooperatively excited [33], thus more Frenkel excitons are formed as the blue shift diminishes with temperature resulting in increased absorption intensities (i.e. increases in $A_{1}$ ). Delocalized interchain CT excitons [47] can be ruled out because the same behavior is observed in both single and double-stranded samples. This leaves the difficulty of explaining intrachain $\mathrm{CT}$ excitons delocalized over several bases. Taken together, we therefore consider the temperature dependence of $\tau_{1}$ as an indication that the FC state is cooperative (as explained in the preceding subsection) and specifically here, that it produces Frenkel excitons delocalized over several bases.

The long-lived component $\tau_{2}$, like the short-lived component $\tau_{1}$, is also qualitatively the same for both single- and double-stranded DNA. However, the attributes of $\tau_{2}$ differ clearly from those of $\tau_{1}$. Most importantly, there is only a rather mild reduction, if any, in the excited state lifetime of the long-lived component. Based on findings that the long decay times in $\mathrm{dA}_{12}$ [40] and poly(A) [33] are nearly identical to those in $\mathrm{dA}_{2}$ and $\mathrm{ApA}$, respectively, it is inferred that only two bases are involved in the long-lived excited state. In contrast to the excited state yielding $\tau_{1}$, where energy is distributed over several bases and where a reduction in lifetime with increasing temperature indicates a reduction in delocalization length, the relatively constant lifetimes of the excited state yielding $\tau_{2}$ indicate that it is less affected by temperature as one would expect for an exciton that involves only two bases. The ESA intensities $\left(\mathrm{A}_{2}\right)$, however, do decrease with increasing temperature and appear to mirror the increase in $\mathrm{A}_{1}$, the latter reflecting an increase in absorption by unstacked bases that cannot relax to the ground state via an excimer. Therefore, we conclude that the nature of the exciton remains unchanged, i.e. it remains an exciton distributed over two bases, but fewer are formed at higher temperatures.

Furthermore, we consider this exciton to have CT character. This inference is consistent with the aforementioned theoretical calculations of polyadenine in water showing an evolution of excitation from the delocalized FC state, via intermonomer charge transfer between two stacked bases, to a dark excimer energy minimum [35]. It is also broadly consistent with the aforementioned model of poly $(\mathrm{dA}) \cdot \operatorname{poly}(\mathrm{dT})$ where intraband scattering causes a reduction in excited state energies, a loss of delocalization length, and an increase in CT character with concomitant increase in lifetime [22]. Finally, it is in agreement with conclusions drawn, from the correlation between experimentally obtained excited state lifetimes and gas phase ionization potentials corrected for gas phase electron affinities, that UV excitation produces exciplex states between two stacked bases [33].

\section{Base Stacking and Base Pairing}

Hybridization in DNA occurs when two complementary strands combine into a double helix through hydrogenbonded base pairing [48]. The close agreement between calorimetric and UV absorption measurements means that the loss of hypochromicity [24, 28] in DNA with increasing temperature can be used as an index of hybridization [48]. Consequently monitoring absorption of a sample at $260 \mathrm{~nm}$ permits determination of its melting temperature [39] - the temperature where only $50 \%$ of the molecules are still hybridized.

Fig. (7) shows a UV melting curve for Sample 3 (Table 1) superimposed on ESA lifetimes, expected to be affected by H-bonding $[49,50]$, for the short-lived excited states $\left(\tau_{1}\right.$, Fig. 7a) as well as the long-lived excited states $\left(\tau_{2}\right.$, Fig. $\left.7 \mathbf{b}\right)$. If base-pairing played a significant role in the efficient deactivation of excited states [49-52], evidence of pronounced changes in ESA attributes occurring near the melting point of the hybridized sample ought to be evident. More specifically, an increase in excited state lifetimes should be observed as the temperature approaches $T_{m}$. There is a lack of correlation between the gain in absorbance, indicative of a loss of H-bonding between base pairs, and the observed excited state lifetimes. In fact, there is, contrary to expectations based on $\mathrm{H}$-bonding deactivation of excited states, a decrease in the observed excited state lifetime $\tau_{1}$. 
(a)

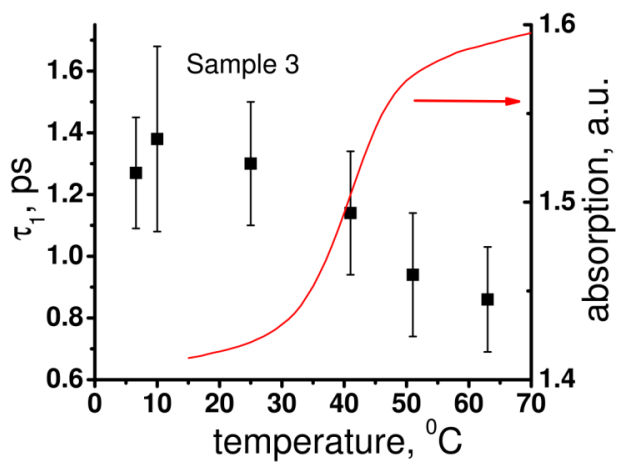

(b)

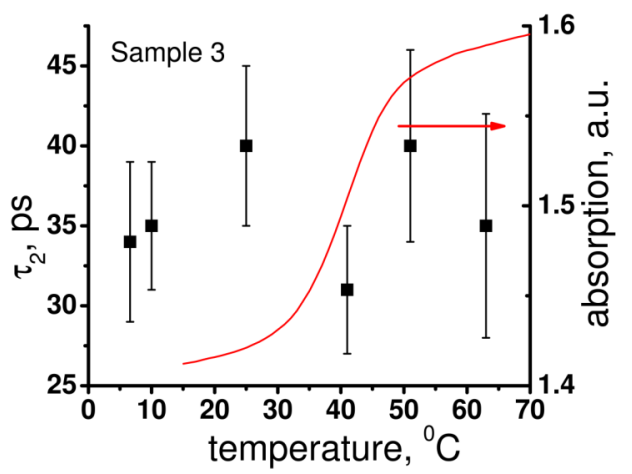

(c)

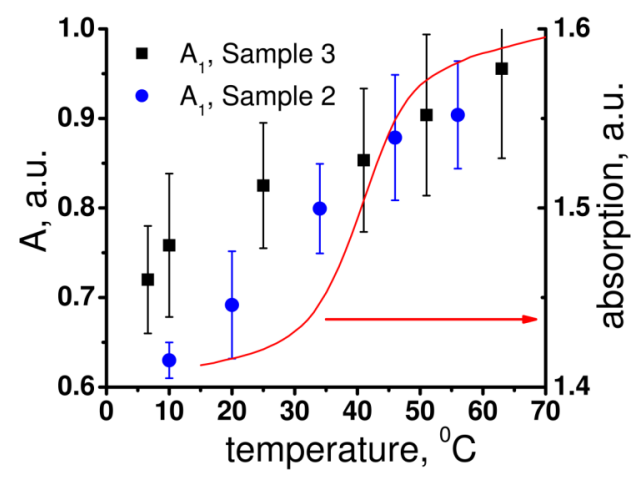

Fig. (7). Further evidence that excited state dynamics in adjacent adenines is dominated by base stacking, but not base pairing, derives from the lack of evidence of melting transitions in both shortlived (a) and long-lived (b) excited state lifetime data for $\left(\mathrm{dA}_{12}\right) \cdot\left(\mathrm{dT}_{12}\right)$. The increase with temperature in the short-lived excited state absorption intensities for double-stranded DNA shows a greater similarity to that of the corresponding single stranded polyadenine sample than to the melting curve (c). The UV absorption melting curve is superimposed in all panels for comparison. Note that the melting temperature is concentration dependent and a small correction $\left(\sim+4{ }^{\circ} \mathrm{C}\right)$ has to be made to align the UV data to the ESA data (UV absorption and ESA sample concentrations were 5 $\mu \mathrm{M}$ and $25 \mu \mathrm{M}$, respectively).

If any changes were to be observed, one may expect ESA intensities to increase with increasing temperature since excited states should persist longer if $\mathrm{H}$-bonding no longer efficiently deactivated them. Although ESA intensities $\left(A_{1}\right)$ increase for the short-lived excited states, it is clear from Fig. (7c) that no evidence of a melting transition, within experimental error, is observed in the double-stranded sample. In fact, Fig. (7c) shows these intensities to be rather more simi- lar to the change in absorption intensities of the short-lived excited states in the corresponding polyadenine single strand. No evidence is found for a melting transition in the absorption intensities of the long-lived excited states $\left(\mathrm{A}_{2}\right.$; data not shown). Indeed, again contrary to expectation based on $\mathrm{H}$ bonding deactivation of excited states, the latter intensities decrease with temperature. Taken together, our results are therefore consistent with a dominant role for base stacking, as opposed to base pairing, affecting electronic excited state relaxation in DNA.

\section{CONCLUSIONS}

Current understanding of excited state dynamics in DNA appears to be converging on a model wherein a UV photon is absorbed collectively by electronically coupled and thus well-stacked intrachain bases. This collective excitation results in a Frenkel exciton that is delocalized over these bases, and the Frenkel exciton then decays rapidly to a long-lived, lower energy, dark intrachain exciplex.

Femtosecond pump-probe $(266 \mathrm{~nm}$ and $400 \mathrm{~nm}$, respectively) transient absorption measurements in DNA and LNA 12-mers of single-stranded and double-stranded oligomers, performed under conditions of increasing temperature to destabilize intrachain base stacking, yielded results that are consistent with this model and thus strengthen it. We observed a reduction in short-lived excited state lifetimes with increasing temperature as would be expected when fewer bases are coupled and available for collective excitation to form Frenkel excitons. We observed an increase in the absorption intensities for these short-lived excited state lifetimes consistent with the expected increase in the fraction of unstacked bases that could absorb UV photons individually. Moreover, at high temperatures, the fast component of the ESA signal approached the excited state decay time of the monomer, hence providing confirmation of absorption by unstacked bases.

We observed no discernible effect of temperature on the long-lived excited state lifetimes. This observation is consistent with the expectation that only two bases are involved in the long-lived excited state and that higher temperatures would disrupt stacks involving more than two bases to a greater extent than stacks of only two bases. A reduction in the absorption intensities of the long-lived states show that fewer of these exist at higher temperatures. This is also consistent with the expectation that they derive from Frenkel excitons of which fewer are formed at elevated temperatures.

A lack of noticeable changes in ESA attributes related to the melting points of the double-stranded samples is indicative of the dominance of stacking, rather than base pairing, in governing the excited state dynamics of those samples probed in this work. This confirms previous observations that the manner of base stacking, and the accompanying degree of coupling between bases, has a strong effect on excited state dynamics $[40,53,54]$. In addition, stacking is also the dominant force imparting structural order to sequences of adjacent adenines, thus the relative lack of differences in excited state dynamics between poly(dA) and poly $(\mathrm{dA})$. $\operatorname{poly}(\mathrm{dT})$.

The emerging model of excited state dynamics reconciles different views regarding single or collective excitation, 
Frenkel or CT exciton formation, and the relative roles of base stacking and base pairing in excited state relaxation. It will provide a point of departure for studies aimed at gaining a more detailed understanding of how these phenomena contribute to the generation of UV-induced photodamage in nucleic acids.

\section{ACKNOWLEDGEMENTS}

Instrumentation and infrastructure were provided by the UBC Laboratory for Advanced Spectroscopy and Imaging Research (LASIR) and Laboratory for Molecular Biophysics (LMB). Funding was provided by the Natural Sciences and Engineering Research Council (NSERC), the Canadian Institutes of Health Research (CIHR), the Canada Foundation for Innovation (CFI) and the British Columbia Knowledge Development Fund (BCKDF). We thank Curtis Hughesman and Yee-Chee Lim for their assistance in preparing the samples and Yee-Chee Lim for obtaining UV absorption measurements.

\section{REFERENCES}

[1] Cantor CR, Schimmel PR. The conformation of biological macromolecules. W. H. Freeman: San Francisco; 1980.

[2] Sponer J, Berger I, Spackova N, Leszczynski J, Hobza P. Aromatic base stacking in DNA: from ab initio calculations to molecular dynamics simulations. J Biomol Struct Dyn 2000; Conversation 11: 383-407.

[3] Rezac J, Hobza P. On the nature of DNA-duplex stability. Chem Eur J 2007; 13: 2983-9.

[4] Luch A. The mode of action of organic carcinogens on cellular structures. In: Bignold LP, Ed. Cancer: cell structures, carcinogens and genomic instability, Birkhauser Verlag, Basel, 2006; pp. 65-95.

[5] Bruskov VI, Malakhova LV, Masalimov ZK, Chernikov AV. Heatinduced formation of reactive oxygen species and 8-oxoguanine, a biomarker of damage to DNA. Nucleic Acids Res 2002; 30: 135463.

[6] Lehoczky P, McHugh PJ, Chovanec M. DNA interstrand cross-link repair in Saccharomyces cerevisiae. FEMS Microbiol Rev 2007; 31: 109-33.

[7] Nejedly K, Kittner R, Pospisilova S, Kypr J. Crosslinking of the complementary strands of DNA by UV light: dependence on the oligonucleotide composition of the UV irradiated DNA. BBA-Gene Struct Expr 2001; 1517: 365-75.

[8] Munger K, Hayakawa H, Nguyen CL, Melquiot NV, Duensing A, Duensing S. Viral carcinogenesis and genomic instability. In: Bignold LP, Ed. Cancer: cell structures, carcinogens and genomic instability, Birkhauser Verlag, Basel, 2006; pp. 179-99.

[9] Kundu LM, Linne U, Marahiel M, Carell T. RNA is more UV resistant than DNA: the formation of UV-induced DNA lesions is strongly sequence and conformation dependent. Chem-Eur J 2004; 10: $5697-705$.

[10] Pattison DI, Davies MJ. Actions of ultraviolet light on cellular structures. In: Bignold LP, Ed. Cancer: cell structures, carcinogens and genomic instability, Birkhauser Verlag, Basel, 2006; pp. 13157.

[11] Sinha RP, Häder D-P. UV-induced DNA damage and repair: a review. Photochem Photobiol Sci 2002; 1: 225-36.

[12] Nix MGD, Devine AL, Cronin B, Ashfold MNR. Ultraviolet photolysis of adenine: dissociation via the ${ }^{1} \pi \sigma^{*}$ state. J Chem Phys 2007; 126: 124312.

[13] Schreier WJ, Schrader TE, Koller FO, et al. Thymine dimerization in DNA is an ultrafast photoreaction. Science 2007; 315: 625-9.

[14] Albinsson B, Norden B. Electronic transition moment directions and identification of low-energy $n \pi *$ states in weakly perturbed purine chromophores. J Am Chem Soc 1993; 115: 223-31.

[15] Holmen A, Broo A, Albinsson B, Norden B. Assignment of electronic transition moment directions of adenine from linear dichroism measurements. J Am Chem Soc 1997; 119: 12240-50.
[16] Hug W, Tinoco I. Electronic spectra of nucleic acid bases. I. Interpretation of in-plane spectra with the aid of all valence electron MO-CI calculations. J Am Chem Soc 1973; 95: 2803-13.

[17] Hug W, Tinoco I. The electronic spectra of nucleic acid bases. II. Out-of-plane transitions and the structure of the nonbonding orbitals. J Am Chem Soc 1974; 96: 665-73.

[18] Preuss M, Schmidt WG, Seino K, Furthmuller J, Bechstedt F. Ground- and excited-state properties of DNA base molecules from plane-wave calculations using ultrasoft pseudopotentials. J Comput Chem 2004; 25: 112-22.

[19] Hobza P, Sponer J. Structure, energetics, and dynamics of the nucleic acid base pairs: nonempirical ab initio calculations. Chem Rev 1999; 99: 3247-76.

[20] Crespo-Hernández CE, Cohen B, Kohler B. Base stacking controls excited state dynamics in A.T DNA. Nature 2005; 436: 1141-4.

[21] Kwok W-M, Ma C, Phillips DL. Femtosecond time- and wavelength-resolved fluorescence and absorption spectroscopic study of the excited states of adenosine and an adenine oligomer. J Am Chem Soc 2006; 128: 11894-905.

[22] Markovitsi D, Gustavsson T, Talbot F. Excited states and energy transfer among DNA bases in double helices. Photochem Photobiol Sci 2007; 6: 717-24.

[23] Parthasarathi R, Subramanian V. Stacking interactions in benzene and cytosine dimers: from molecular electron density perspective. Struct Chem 2005; 16: 243-55.

[24] Rhodes W. Hypochromism and other spectral properties of helical polynucleotides. J Am Chem Soc 1961; 83: 3609-17.

[25] Rich A, Kasha M. The $\mathrm{n} \rightarrow \pi^{*}$ transition in nucleic acids and polynucleotides. J Am Chem Soc 1960; 82: 6197-9.

[26] Sponer J, Leszczynski J, Hobza P. Nature of nucleic acid-base stacking: nonempirical ab initio and empirical potential characterization of 10 stacked base dimers. Comparison of stacked and Hbonded base pairs. J Phys Chem 1996; 100: 5590-6.

[27] Sponer J, Leszczynski J, Hobza P. Hydrogen bonding, stacking and cation binding of DNA bases. J Mol Struct 2001; 573: 43-53.

[28] Tinoco I. Hypochromism in polynucleotides. J Am Chem Soc 1960; 82: 4785-90

[29] Wilson RW, Callis PR. Excitons, energy transfer, and charge resonance in excited dinucleotides and polynucleotides. A photoselection study. J Phys Chem 1976; 80: 2280-8.

[30] Hu L-H, Zhao Y, Wang F, et al. Are adenine strands helical Haggregates? J Phys Chem B 2007; 111: 11812-6.

[31] Kabelac M, Ryjacek F, Hobza P. Already two water molecules change planar H-bonded structures of the adenine-thymine base pair to the stacked ones: a molecular dynamics simulations study. Phys Chem Chem Phys 2000; 2: 4906-9.

[32] Buchvarov I, Wang Q, Raytchev M, Trifonov A, Fiebig T. Electronic energy delocalization and dissipation in single- and doublestranded DNA. Proc Natl Acad Sci USA 2007; 104: 4794-7.

[33] Takaya T, Su C, de La Harpe K, Crespo-Hernández CE, Kohler B. UV excitation of single DNA and RNA strands produces high yields of exciplex states between two stacked bases. Proc Natl Acad Sci USA 2008; 105: 10285-90.

[34] Crespo-Hernández C, de La Harpe K, Kohler B. Ground-state recovery following UV excitation is much slower in G.C-DNA duplexes and hairpins than in mononucleotides. J Am Chem Soc 2008; 130: 10844-45.

[35] Santoro F, Barone V, Improta R. Influence of base stacking on excited-state behavior of polyadenine in water, based on timedependent density functional calculations. Proc Natl Acad Sci USA 2007; 104: 9931-6.

[36] Bittner ER. Frenkel exciton model of ultrafast excited state dynamics in AT DNA double helices. J Photochem Photobiol A 2007; 190: 328-34.

[37] Bouvier B, Dognon J-P, Lavery R, et al. Influence of conformational dynamics on the exciton states of DNA oligomers. J Phys Chem B 2003; 107: 13512-22.

[38] Nielsen KE, Rasmussen J, Kumar R, Wengel J, Jacobsen JP, Petersen M. NMR studies of fully modified locked nucleic acid (LNA) hybrids: solution structure of an LNA:RNA hybrid and characterizartion of LNA:DNA hybrid. Bioconjug Chem 2004; 15: 449-57.

[39] SantaLucia J, Allawi HT, Seneviratne PA. Improved nearestneighbor parameters for predicting DNA duplex stability. Biochem-US 1996; 35: 3555-62.

[40] Konorov SO, Schulze HG, Addison CJ, Haynes CA, Blades MW, Turner RFB. Base stacking configuration is a major determinant of 
excited state dynamics in A.T DNA and LNA. Open Spectrose J 2008; 3: 9-20.

[41] Alexeev DG, Lipanov AA, Skuratovskii IY. Poly(dA) poly(dT) is a $B$-type double helix with a distinctively narrow minor groove. Nature 1987; 325: 821-3.

[42] Premilat S, Albiser G. X-Ray fibre diffraction study of an elevated temperature structure of poly(dA)poly(dT). J Mol Biol 1997; 274: 64-71.

[43] Crespo-Hernández C, Kohler B. Influence of secondary structure on electronic energy relaxation in adenine homopolymers. J Phys Chem B 2004; 108: 11182-8.

[44] Dewey TG, Turner DH. Laser temperature-jump study of stacking in adenylic acid polymers. Biochem-US 1979; 18: 5757-62.

[45] Emanuele E, Markovitsi D, Millie P, Zakrzewksa K. UV spectra and excitation delocalization in DNA: influence of the spectral width. Chem Phys Chem 2005; 6: 1387-92.

[46] Dubovsky C, Mukamel S. Charge-transfer excitons and $\chi^{(2)}$ of molecular monolayers. J Chem Phys 1992; 96: 9201-11.

[47] Conwell EM, McLaughlin PM, Bloch SM. Charge-transfer excitons in DNA. J Phys Chem B 2008; 112: 2268-72.
[48] Saenger W. Principles of nucleic acid structure. Springer: New York; 1984.

[49] Schultz T, Samoylova E, Radloff W, Hertel IV, Sobolewski AL, Domcke W. Efficient deactivation of a model base pair via excitedstate hydrogen transfer. Science 2004; 306: 1765-8.

[50] Schwalb NK, Temps F. Ultrafast electronic relaxation in guanosine is promoted by hydrogen bonding with cytidine. J Am Chem Soc 2007; 129: 9272-3.

[51] Lami A, Villani G. Non-stationary states in chemistry. Theor Chem Acc 2007; 117: 755-64.

[52] Reece SY, Hodgkiss JM, Stubbe J, Nocera DG. Proton-coupled electron transfer: the mechanistic underpinning for radical transport and catalysis in biology. Philos T Roy Soc B 2006; 361: 1351-64.

[53] Cohen B, Larson MH, Kohler B. Ultrafast excited-state dynamics of RNA and DNA C tracts. Chem Phys 2008; 350: 165-74.

[54] Hatcher E, Balaeff A, Keinan S, Venkatramani R, Beratan DN. PNA versus DNA: effects of structural fluctuations on electronic structure and hole-transport mechanisms. J Am Chem Soc 2008; 130: $11752-61$.

(C) Konorov et al.; Licensee Bentham Open

This is an open access article licensed under the terms of the Creative Commons Attribution Non-Commercial License (http://creativecommons.org/licenses/ by-nc/3.0/) which permits unrestricted, non-commercial use, distribution and reproduction in any medium, provided the work is properly cited. 